Research Paper

\title{
Patients with Gastric Polyps need Colonoscopy Screening at Younger Age: A Large Prospective Cross-Sectional Study in China
}

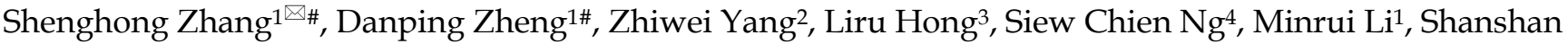 \\ Huang ${ }^{1}$, Shengbing Wang ${ }^{2}$, Li Li ${ }^{1}$, Manying Li ${ }^{1}$, Hongshi Zhang ${ }^{3}$, Jinghua Lin ${ }^{1}$, Bihui Zhong1, Yi Cui ${ }^{\bowtie}$, \\ Minhu Chen ${ }^{1 凶}$ \\ 1. Division of Gastroenterology, The First Affiliated Hospital, Sun Yat-sen University, Guangzhou, P.R. China \\ 2. Division of Gastroenterology, Meizhou People's Hospital, Meizhou, P.R. China \\ 3. Division of Gastroenterology, Shantou Central Hospital, Shantou, P.R. China \\ 4. Department of Medicine and Therapeutics, State Key Laboratory of Digestive Disease, Institute of Digestive Disease, Li Ka Shing Institute of Health Science, \\ Hong Kong, P.R. China \\ \# These authors contributed equally to the work \\ $\triangle$ Corresponding authors: Shenghong Zhang, M.D., Ph.D., NO. 58, Zhongshan Road 2, Guangzhou, P. R. China, E-mail: shenghongzhang@163.com. or Minhu \\ Chen, M.D., Ph.D., NO. 58, Zhongshan Road 2, Guangzhou, P. R. China, E-mail: chenminhu@mail.sysu.edu.cn or Yi Cui, M.D., NO. 58, Zhongshan Road 2, \\ Guangzhou, P. R. China, E-mail: gzcuiyi@163.com
}

(c) The author(s). This is an open access article distributed under the terms of the Creative Commons Attribution License (https://creativecommons.org/licenses/by/4.0/). See http://ivyspring.com/terms for full terms and conditions.

Received: 2019.01.06; Accepted: 2019.06.23; Published: 2019.08.07

\begin{abstract}
Background To date, it is not clarified whether patients with gastric polyps without any alarming symptoms for colorectal neoplasia need colonoscopy screening. The objective of this study is to prospectively determine the association between gastric polyps and colorectal neoplasia.

Methods A multicenter prospective cross-sectional study was performed from July 2012 to December 2014. We compared patients with and without gastric polyps for prevalence of colorectal adenomas. The odds ratios (OR) were computed by logistic regression analysis after multivariable adjustments.

Results Totally 1546 patients were included, with 770 patients in the gastric polyp group and 776 in the ageand sex- matched control group. Patients with gastric polyps had greater odds of having any colorectal adenoma (adjusted $\mathrm{OR}=2.34,95 \%$ confidence interval $[\mathrm{Cl}]: 1.79$ to $3.06, \mathrm{p}<0.001$ ) and advanced colorectal adenomas (adjusted OR=2.71, 95\% Cl: 1.74 to $4.23, \mathrm{p}<0.001$ ) than those without. The positive association between gastric polyps and colorectal adenomas remained significant in both women (OR=2.34, 95\% Cl: 1.66 to $3.29, p<0.001)$ and men $(O R=1.87,95 \% \mathrm{Cl}: 1.31$ to $2.66, p=0.001)$. Patients over the age of 40 with gastric polyps had a higher prevalence of colorectal adenomas than those without $(40-49 y \mathrm{y}:$ OR $=1.81,95 \%$ $\mathrm{Cl}=1.02-3.21, \mathrm{p}=0.04 ; 50-59 y \mathrm{y}: \mathrm{OR}=1.88,95 \% \mathrm{Cl}=1.26-2.81, \mathrm{p}<0.001 ; 60-74 \mathrm{yr}: \mathrm{OR}=2.62,95 \% \mathrm{Cl}=1.73-3.98$, $\mathrm{p}<0.001)$.

Conclusions The presence of gastric polyps is significantly associated with a higher prevalence of colorectal adenomas, especially advanced colorectal adenomas. Colonoscopy might be considered in patients with gastric polyps, of any gender, and over the age of 40 .
\end{abstract}

Key words: gastric polyp; colonoscopy; colorectal adenoma; colorectal cancer

\section{Background}

Colonoscopy is widely accepted as a primary tool in screening for colorectal cancer (CRC) and precancerous polyps $[1,2]$. Colonoscopic polypectomy is associated with a $53 \%$ reduction in the long-term mortality of CRC [3]. Current national guidelines recommend $\mathrm{CRC}$ screening in average-risk asymptomatic individuals aged more than 50 to prevent cancer incidence and mortality [4, 5]. In countries with limited resources, it is important to risk-stratify patients for CRC screening. Risk factors for CRC and advanced adenomas have been identified, including age, sex, smoking, and family 
history [6]. Additionally, colorectal neoplasms occur more frequently in patients with gastric cancer $[7,8]$. However, detection of colorectal neoplasms in gastric cancer patients might not change the prognosis greatly, especially in late stage of cancer. Thus, it is important to know whether individuals with sporadic gastric polyps would also benefit from screening colonoscopy by detecting synchronous or metachronous colorectal neoplasms.

To date, available knowledge regarding this issue is limited and has been gathered in a meta-analysis. ${ }^{9}$ The combined analysis of five heterogeneous retrospective studies suggested that the prevalence of colorectal neoplasms was 1.15 to 1.31 times higher in patients with gastric polyps than those without, focusing mainly on fundic gland polyps (FGP) [9]. However, these retrospective studies were unable to differentiate various confounding factors that influenced the association. Selection bias was inevitably introduced, making the conclusions less convincing. Moreover, whether the associations between gastric polyps and colorectal adenomas differ by age and sex stratifications is not clarified yet. Although one study has shown that the association between gastric polyps and colorectal polyps remained positive after gender stratification [10], another study only found a positive association in women [11]. Besides, very few studies addressed the outcome of advanced colorectal adenomas, despite only one case-control study with small sample size [12], showing no significant association between gastric polyps and the advanced colorectal adenomas.

Therefore, the current evidence regarding the association between sporadic gastric polyps and colorectal neoplasia remains scarce, mainly based on restricted-sized retrospective studies. To address this issue, we conducted a large multicenter prospective cross-sectional study to investigate the potential relationship between the presence of gastric polyps and prevalence of colorectal adenomas.

\section{Methods}

\section{Study design and participants}

We performed a cross-sectional study (ChiCTR--OCC-12003010) by matching at-risk subjects (those with gastric polyps) and disease-free subjects (those without gastric polyps) and determining the likelihood that these patients had colorectal adenomas, including advanced colorectal adenomas. This multicenter study was conducted in the First Affiliated Hospital of Sun Yat-sen University, Shantou Central Hospital, and Meizhou People's Hospital. Consecutive subjects who underwent esophagogastroduodenoscopy (EGD) were prospectively enrolled between July 2012 and December 2014. Participants in the gastric polyp group had different types of polyps, detected by EGD and confirmed by pathology, including FGP, gastric hyperplastic polyps, adenomas, and inflammatory polyps. The control group comprised of sex- and age-matched participants without detection of gastric polyps by EGD during the same period, randomly selected using a one-to-one matching approach. Both groups of patients underwent total colonoscopy at the same day or within six months of the EGD procedure. Participants were included if they were between the ages of 18 and 74, and excluded according to the following criteria: 1) having a history of gastrointestinal tumors or colorectal polyps; 2) having a history of gastrointestinal surgery, including colorectal polypectomy; 3) having a history of inflammatory bowel disease, intestinal tuberculosis, or familial colorectal polyposis syndrome; 4) presenting with alarm symptoms of CRC, including hematochezia, positive fecal occult blood test, recent bowel habit changes, significant weight loss, or anemia; 5) failing to undergo colonoscopy within six months after EGD; 6) technically unsatisfactory colonoscopy owing to poor or inadequate bowel preparation or incomplete colonoscopy; and 7) with gastric polyps that were histologically confirmed as carcinoid tumors, lymphomas, fibromas, lipomas, leiomyomas, ectopic pancreatic tissues, or normal tissue.

The study was conducted and reported according to the study protocol, conforming to the ethical guidelines of the 1975 Declaration of Helsinki, which was approved by Ethics Review Committees at each study center on 28 June, 2012. Written informed consents were obtained from participants upon their arrival at the study clinic and before the procedures.

\section{Endoscopic procedure and pathological examinations}

At each participating center, EGD and colonoscopy procedures were performed by experienced gastroscopists and colonoscopists respectively, who had performed at least 2000 gastroscopies, 1000 colonoscopies, and 200 polypectomies. Patients were prescribed two liters of polyethylene glycol oral lavage for bowel preparation 4 to 6 hours before the colonoscopy procedure. All polyps detected during EGD and colonoscopy were recorded, including the number, size, and location. Polyp size was estimated endoscopically using open biopsy forceps, or measured following specimen resection. Gastric polyps were classified according to their location in the fundus, body, antrum, cardia, or pylorus. The locations of colorectal polyps were 
categorized as right colon (proximal to splenic flexure or a colonoscopic insertion depth of more than $55 \mathrm{~cm}$ ), left colon (distal to splenic flexure or an insertion depth of less than $55 \mathrm{~cm}$ ), or both right and left colon, as previously reported [13]. The pathology of all resected colorectal polyps was evaluated by certified gastrointestinal pathologists, classified as non-neoplastic (hyperplastic or inflammatory polyps) or neoplastic (adenomatous). Adenomatous polyps were categorized as tubular, tubulovillous, or villous adenomas according to the World Health Organization criteria [14]. Gastroscopists, colonoscopists and pathologists were blinded to each other's findings.

\section{Outcome and variable assessment}

The primary outcome was the association between the presence of gastric polyps and the prevalence of colorectal adenomas. The secondary outcome was the prevalence of advanced colorectal adenomas among subjects with gastric polyps compared to those without. We also sought to determine whether these associations differed when patients were stratified by sex and age. Patients with advanced adenomas were classified as having at least one adenoma with at least one of the following characteristics: size of $\geq 10 \mathrm{~mm}$ in diameter, tubulovillous or villous components, or high-grade dysplasia [15]. Pathological diagnosis of intramucosal carcinoma or carcinoma in situ was classified as high-grade dysplasia [16]. CRC was graded according to the TNM staging system [17].

Data regarding baseline characteristics of the participants were collected, including age, sex, body mass index (BMI), educational level, symptoms, family history of CRC, current smoking ( $\geq 1$ cigarette per day for more than one year), alcohol consumption (at least $70 \mathrm{~g}$ per week), non-steroidal anti-inflammatory drugs (NSAIDS)/aspirin use (for at least three months) [18], history of hypertension (systolic pressure $\geq 140 \mathrm{mmHg}$ and/or diastolic $\geq 90$ $\mathrm{mmHg})$, fasting hyperglycemia ( $\geq 7.0 \mathrm{mmol} / \mathrm{L})$, hepatic steatosis (detected by abdominal ultrasonography), and dyslipidemia. Blood glucose and lipid profiles were measured following eight hours of fasting. Helicobacter pylori (H. pylori) infection was checked by urea breath tests or urease tests.

\section{Statistical analysis}

Symmetrically distributed continuous variables were presented as means with standard deviations, while categorical variables were presented as frequencies and percentages. To compare the baseline characteristics of participants in each group, we used the Pearson's chi-squared test for analysis of discrete variables and a t-test for continuous variables. We examined the association between gastric polyps and colorectal adenomas using binary logistic regression analysis. Adjusted odds ratios (OR) and their corresponding 95\% confidence intervals (CI) were estimated by multivariable adjustments of baseline factors. In addition, we evaluated the relationship between gastric polyps and colorectal adenomas according to the number, size, location, and histopathology of both gastric polyps and colorectal adenomas, using both unadjusted and adjusted ORs and their corresponding 95\% CIs. We also compared the presence of colorectal adenomas in patients with and without gastric polyps after stratifying by age and sex.

Pre-specified subgroup analysis was performed based on baseline covariates. Whether the prevalence of colorectal adenoma differed in certain pre-specified subgroups was assessed by testing the interaction effect between gastric polyps and subgroups with the use of logistic models.

All statistical analyses were performed using the Statistical Product and Service Solutions (SPSS) software, version 22.0 (IBM Corp, Armonk, NY, USA). All two-tailed tests were regarded as significant with a $p$ value of less than 0.05 .

\section{Results}

\section{Baseline characteristics of the study}

A detailed participant enrollment process is presented in Figure 1. After excluding patients who failed to undergo colonoscopy within six months after EGD and patients with a technically unsatisfactory colonoscopy, 800 subjects with different types of gastric polyps, detected by EGD and confirmed by pathology, were enrolled according to the inclusion criteria. During the same time period, 800 sex- and age-matched gastric polyp-free control patients were enrolled. The indications for EGD included abdominal pain, gastroesophageal reflux, dyspepsia, upper gastrointestinal bleeding, and screening. After the application of strict exclusions, 1546 individuals were finally included, with 770 in the gastric polyp group and 776 in the gastric polyp-free control group.

Baseline characteristics were compared between patients with and without gastric polyps and the results were shown in Table 1. Subjects in these two groups did not differ significantly in age, sex, BMI, education level, positive CRC family history, hypertension, fasting hyperglycemia, steatosis, or potential use of chemopreventive agents, including NSAIDS and aspirin. However, significantly fewer subjects in the gastric polyp group were symptomatic (including abdominal pain, dyspepsia, and 
gastroesophageal reflux), had current smoking, alcohol consumption, and positive $H$. pylori infection than those in the gastric polyp-free group. Lipid profiles differed significantly between the two groups, except for triglyceride levels.

Table 1 Comparison of baseline characteristics between subjects with and without gastric polyps

\begin{tabular}{|c|c|c|c|}
\hline Characteristics & $\begin{array}{l}\text { Non-gastric polyps } \\
(\mathrm{n}=776)\end{array}$ & $\begin{array}{l}\text { Gastric polyps } \\
(\mathrm{n}=770)\end{array}$ & $P$ value \\
\hline Age (years) & $52.4 \pm 11.3$ & $52.6 \pm 11.5$ & 0.67 \\
\hline Sex (\% male) & $302(38.9 \%)$ & $289(37.5 \%)$ & 0.58 \\
\hline College graduate $(\%)$ & $95(12.2 \%)$ & $93(12.1 \%)$ & 0.92 \\
\hline $\mathrm{BMI} \geq 25 \mathrm{~kg} / \mathrm{m}^{2}(\%)$ & $130(16.8 \%)$ & $132(17.1 \%)$ & 0.84 \\
\hline Family history of CRCs (\%) & $27(3.5 \%)$ & $33(4.3 \%)$ & 0.41 \\
\hline Current smoking (\%) & $103(13.3 \%)$ & $51(6.6 \%)$ & $<0.001$ \\
\hline Alcohol consumption (\%) & $50(6.4 \%)$ & $22(2.9 \%)$ & 0.001 \\
\hline History of hypertension (\%) & $100(12.9 \%)$ & $115(14.9 \%)$ & 0.24 \\
\hline NSAIDS/aspirin use (\%) & $18(2.3 \%)$ & $22(2.9 \%)$ & 0.51 \\
\hline $\begin{array}{l}\text { Presenting with symptoms } \\
(\%) *\end{array}$ & $599(77.2 \%)$ & $543(70.5 \%)$ & 0.003 \\
\hline $\begin{array}{l}\text { Presence of hepatic steatosis } \\
(\%) \dagger\end{array}$ & $117(15.1 \%)$ & $114(14.8 \%)$ & 0.88 \\
\hline $\begin{array}{l}\text { Positive H. pylori infection } \\
(\%)\end{array}$ & $369(47.6 \%)$ & $250(32.5 \%)$ & $<0.001$ \\
\hline \multicolumn{4}{|l|}{$\begin{array}{l}\text { Lipid profiles and blood } \\
\text { glucose }\end{array}$} \\
\hline Triglyceride (mmol/L) & $1.31 \pm 1.15$ & $1.35 \pm 0.96$ & 0.45 \\
\hline Total cholesterol (mmol/L) & $4.69 \pm 1.40$ & $5.15 \pm 1.12$ & $<0.001$ \\
\hline LDL cholesterol (mmol/L) & $2.56 \pm 0.96$ & $3.03 \pm 0.96$ & $<0.001$ \\
\hline HDL cholesterol (mmol/L) & $1.32 \pm 0.40$ & $1.43 \pm 0.36$ & $<0.001$ \\
\hline $\begin{array}{l}\text { Fasting hyperglycemia } \\
(\geq 7.0 \mathrm{mmol} / \mathrm{L})(\%)\end{array}$ & $61(7.9 \%)$ & $42(5.5 \%)$ & 0.06 \\
\hline
\end{tabular}

* Patients with alarm symptoms of CRC (including hematochezia, positive fecal occult blood test, recent bowel habit changes, significant weight loss, or anemia) were excluded from this study; patients with symptoms of abdominal pain, dyspepsia and gastroesophageal reflux were included. $†$ The presence of hepatic steatosis was detected by abdominal ultrasonography. BMI-body mass index; CRC-colorectal cancer; H. pylori-helicobacter pylori; NSAIDS-non-steroidal anti-inflammatory drugs; LDL- low density lipoprotein; HDL-high density lipoprotein.

\section{Association of gastric polyps with colorectal adenomas}

During the study period, we detected colorectal polyps in 506 participants by colonoscopy, including 198 of the 776 patients $(25.5 \%)$ who did not have gastric polyps, and 308 of the 770 patients (40.0\%) who did. Among the control group, we identified 133 subjects $(17.1 \%)$ with any type of colorectal adenoma, of which $35(4.5 \%)$ were classified as having advanced colorectal adenomas. In the gastric polyp group, 229 subjects $(29.7 \%)$ had any type of colorectal adenoma, of which $77(10.0 \%)$ had advanced colorectal adenomas. Table 2 presents the ORs of gastric polyps and baseline factors for colorectal adenomas as well as advanced colorectal adenomas. After multivariable adjustments, we found that subjects with gastric polyps had greater odds of having any colorectal adenoma (adjusted OR=2.34, 95\% CI: 1.79 to 3.06, $\mathrm{p}<0.001)$ as well as advanced colorectal adenomas (adjusted OR=2.71, 95\% CI: 1.74 to $4.23, \mathrm{p}<0.001$ ) compared with those in the control group. The adjusted ORs of gastric polyps for colorectal adenomas in the First Affiliated Hospital of Sun Yat-sen University, Shantou Central Hospital, and Meizhou People's Hospital were 3.56 (95\% CI: 2.41 to 5.26, p<0.001), 2.35 (95\% CI: 1.03 to 5.37, $\mathrm{p}=0.04)$ and 2.65 (95\% CI: 1.08 to $6.53, \mathrm{p}=0.03$ ), respectively.

\section{Association of gastric polyps with different types of colorectal neoplasia}

Compared with the control group, patients with gastric polyps had significantly increased prevalence of colorectal adenomas when colorectal adenomas were stratified by number, location, size, and histopathology (Table 3). Besides adenomas, we found no difference in the prevalence of colorectal hyperplastic polyps (adjusted OR: 0.99, 95\% CI: 0.67 to 1.47, $\mathrm{p}=0.96$ ) between patients with and without gastric polyps. Furthermore, we found that the prevalence of CRC was $1.3 \%(10 / 776)$ in the gastric polyp group and $0.3 \%(2 / 770)$ in the control group, with an adjusted OR of 7.05 (95\% CI: 1.35 to 36.81, $\mathrm{p}=0.02$ ) (Detailed characteristics of patients with CRC were shown in Supplemental Table 1). In the gastric polyp group, six early-stage adenocarcinomas were detected and removed endoscopically. Additionally, we identified serrated adenomas in one patient $(1 / 776,0.1 \%)$ in the control group and two patients $(2 / 770,0.3 \%)$ in the gastric polyp group.

\section{Association between gastric polyp characteristics and colorectal adenomas}

Patients with gastric polyps had significantly higher prevalence of colorectal adenoma and advanced adenomas, when stratified by the number, distribution, size, histopathology of gastric polyps, and whether H. pylori infection was detected (Table 4). However, we found no significant correlation between gastric inflammatory polyps and the detection of advanced colorectal adenomas (adjusted $\mathrm{OR}=0.79,95 \%$ CI: 0.24 to $2.64, \mathrm{p}=0.49$ ).

\section{Association between gastric polyps and colorectal adenomas stratified by sex and age}

Women with gastric polyps had higher prevalence of any colorectal adenoma (OR=2.34, 95\% CI: 1.66 to $3.29, \mathrm{p}<0.001)$ as well as advanced colorectal adenomas (OR=4.31, 95\% CI: 2.05 to 9.02, $\mathrm{p}<0.001)$ compared with those without gastric polyps. Such significant associations remained in any type of colorectal neoplasia when FGP and gastric hyperplastic polyps were analyzed separately (Table 5). For men, subjects with gastric polyps had higher prevalence of any colorectal adenoma (OR=1.87, 95\% CI: 1.31 to $2.66, p=0.001$ ) and advanced colorectal 
adenomas (OR=1.71, 95\% CI: 1.01 to $2.88, \mathrm{p}=0.04)$. However, the associations of gastric polyps with CRC were not significant in men (OR=1.57, 95\% CI: 0.26 to 9.49, p=0.62), mainly due to limited cases of CRC. Furthermore, we found no significant association between gastric hyperplastic polyps and the prevalence of any colorectal adenoma type in men (Table 5).

Patients over the age of 40 with gastric polyps were associated with a higher prevalence of any adenoma (40-49yr: OR=1.81, 95\% CI=1.02-3.21, $\mathrm{p}=0.04 ;$ 50-59yr: OR=1.88, 95\% CI=1.26-2.81, $\mathrm{p}<0.001$; 60-74yr: OR=2.62, 95\% CI=1.73-3.98, p<0.001) and advanced adenomas (40-49yr: OR $=2.87, \quad 95 \%$ $\mathrm{CI}=1.10-7.45, \quad \mathrm{p}=0.03 ; \quad 50-59 \mathrm{yr}: \quad \mathrm{OR}=1.93, \quad 95 \%$ $\mathrm{CI}=0.98-3.79, \quad \mathrm{p}=0.05 ; \quad 60-74 \mathrm{yr}: \quad \mathrm{OR}=2.17, \quad 95 \%$ $\mathrm{CI}=1.11-4.23, \mathrm{p}=0.02)$. The association remained significant in patients with FGP. In patients with gastric hyperplastic polyps over the age of 50 , we detected a significantly higher rate of colorectal adenomas. In subjects under 40 years of age, no significant associations between gastric polyps and colorectal adenomas were detected (Table 6).

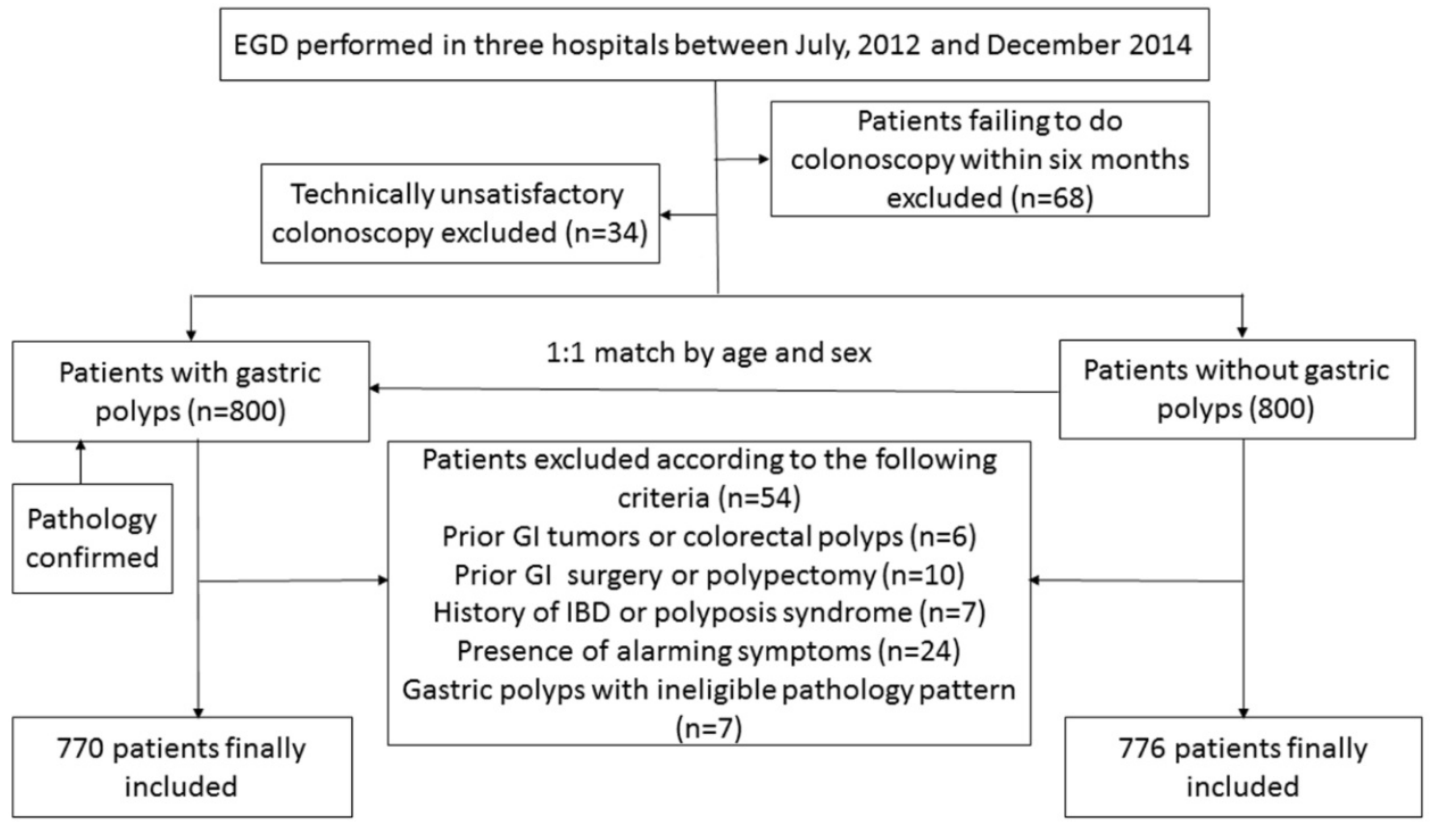

Figure 1. Diagram of enrollment in study groups. EGD: esophagogastroduodenoscopy; GI: gastrointestinal; IBD: inflammatory bowel disease.

Table 2 Multivariable analyses on odds ratios of baseline characteristics for colorectal neoplasia

\begin{tabular}{|c|c|c|c|c|}
\hline \multirow[t]{3}{*}{ Variables } & \multicolumn{2}{|l|}{ Any adenoma } & \multicolumn{2}{|l|}{ Advanced adenoma } \\
\hline & Multivariable analysis & & Multivariable analysis & \\
\hline & OR $(95 \% \mathrm{CI})$ & P value & OR $(95 \% \mathrm{CI})$ & P value \\
\hline Male gender & $1.65(1.26,2.16)$ & $<0.001$ & $1.98(1.28,3.05)$ & 0.002 \\
\hline Age, year & $1.04(1.03,1.05)$ & $<0.001$ & $1.03(1.01,1.05)$ & 0.002 \\
\hline BMI, $\mathrm{kg} / \mathrm{m}^{2}$ & $1.02(0.98,1.07)$ & 0.36 & $1.05(0.98,1.13)$ & 0.17 \\
\hline College graduate & $0.88(0.59,1.31)$ & 0.52 & $0.55(0.26,1.17)$ & 0.12 \\
\hline CRC Family history & $1.94(1.06,3.54)$ & 0.03 & $2.47(1.08,5.66)$ & 0.03 \\
\hline Current smoking & $2.33(1.52,3.58)$ & $<0.001$ & $1.97(1.06,3.67)$ & 0.03 \\
\hline Alcohol consumption & $1.08(0.60,1.95)$ & 0.80 & $1.16(0.49,2.75)$ & 0.74 \\
\hline History of hypertension & $1.44(1.02,2.03)$ & 0.04 & $0.84(0.47,1.50)$ & 0.56 \\
\hline NSAIDS/aspirin use & $0.44(0.19,1.02)$ & 0.06 & $0.61(0.17,2.17)$ & 0.45 \\
\hline Symptomatic & $0.73(0.55,0.97)$ & 0.03 & $0.77(0.50,1.19)$ & 0.24 \\
\hline Presence of steatosis & $1.17(0.81,1.69)$ & 0.40 & $0.91(0.50,1.63)$ & 0.74 \\
\hline Positive H. pylori infection & $1.19(0.92,1.55)$ & 0.19 & $0.92(0.60,1.40)$ & 0.69 \\
\hline Fasting glycemia $\geq 7.0 \mathrm{mmol} / \mathrm{L}$ & $1.06(0.65,1.72)$ & 0.83 & $1.52(0.75,3.05)$ & 0.25 \\
\hline Triglyceride $>1.70 \mathrm{mmol} / \mathrm{L}$ & $0.88(0.63,1.23)$ & 0.46 & $1.09(0.65,1.82)$ & 0.75 \\
\hline Total cholesterol $>5.70 \mathrm{mmol} / \mathrm{L}$ & $1.20(0.81,1.77)$ & 0.36 & $1.01(0.54,1.89)$ & 0.98 \\
\hline $\mathrm{LDL}>3.61 \mathrm{mmol} / \mathrm{L}$ & $1.17(0.79,1.74)$ & 0.43 & $0.79(0.42,1.50)$ & 0.47 \\
\hline $\mathrm{HDL}<1.09 \mathrm{mmol} / \mathrm{L}$ & $1.31(0.95,1.82)$ & 0.10 & $1.63(1.00,2.65)$ & 0.05 \\
\hline Gastric polyps & $2.34(1.79,3.06)$ & $<0.001$ & $2.71(1.74,4.23)$ & $<0.001$ \\
\hline
\end{tabular}

BMI-body mass index; CRC-colorectal cancer; H. pylori-helicobacter pylori; NSAIDS-non-steroidal anti-inflammatory drugs; LDL- low density lipoprotein; HDL-high density lipoprotein; OR-odds ratio; CI-confidence interval. 
Table 3 Unadjusted and adjusted odds ratios for the prevalence of colorectal neoplasia stratified by different types

\begin{tabular}{|c|c|c|c|c|c|c|}
\hline \multirow{2}{*}{$\begin{array}{l}\text { Characteristics of } \\
\text { colorectal polyps }\end{array}$} & \multirow[t]{2}{*}{ No. in non-gastric polyp group (\%) } & \multirow[t]{2}{*}{ No. in gastric polyp group (\%) } & \multicolumn{2}{|l|}{ Unadjusted } & \multicolumn{2}{|l|}{ Adjusted } \\
\hline & & & OR $(95 \% \mathrm{CI})$ & P value & OR $(95 \% \mathrm{CI})$ & P value \\
\hline \multicolumn{7}{|l|}{ Adenoma } \\
\hline \multicolumn{7}{|l|}{ Number } \\
\hline Single & $62(8.0 \%)$ & $111(14.4 \%)$ & $1.94(1.40,2,69)$ & $<0.001$ & $1.91(1.37,2.66)$ & $<0.001$ \\
\hline Multiple ( $\geq 2$ ) & $71(9.1 \%)$ & $118(15.3 \%)$ & $1.80(1.31,2,46)$ & $<0.001$ & $2.16(1.54,3.01)$ & $<0.001$ \\
\hline \multicolumn{7}{|l|}{ Distribution } \\
\hline Right colon & $36(4.6 \%)$ & $88(11.4 \%)$ & $2.65(1.78,3.96)$ & $<0.001$ & $2.61(1.74,3.92)$ & $<0.001$ \\
\hline Left colon & $57(7.3 \%)$ & $147(19.1 \%)$ & $2.98(2.15,4.12)$ & $<0.001$ & $3.16(2.27,4.39)$ & $<0.001$ \\
\hline Both & $40(5.2 \%)$ & $73(9.5 \%)$ & $1.93(1.29,2.87)$ & 0.001 & $1.95(1,29,2.93)$ & 0.001 \\
\hline \multicolumn{7}{|l|}{ Largest polyp size } \\
\hline$<5 \mathrm{~mm}$ & $36(4.6 \%)$ & $59(7.7 \%)$ & $1.71(1.11,2.62)$ & 0.013 & $1.68(1,08,2.62)$ & 0.021 \\
\hline $5-9 \mathrm{~mm}$ & $68(8.4 \%)$ & $104(13.5 \%)$ & $1.71(1.23,2.37)$ & 0.001 & $1.76(1,26,2.47)$ & 0.001 \\
\hline$\geq 10 \mathrm{~mm}$ & $32(4.1 \%)$ & $66(8.6 \%)$ & $2.18(1.41,3.37)$ & $<0.001$ & $2.37(1,52,3.70)$ & $<0.001$ \\
\hline \multicolumn{7}{|c|}{ Histology (other than advanced adenoma) } \\
\hline Tubular adenoma & $118(15.2 \%)$ & $203(26.4 \%)$ & $2.00(1.55,2.57)$ & $<0.001$ & $2.17(1.65,2.85)$ & $<0.001$ \\
\hline $\begin{array}{l}\text { Tubulovillous/villous } \\
\text { adenoma }\end{array}$ & $21(2.7 \%)$ & $40(5.2 \%)$ & $1.97(1.15,3.37)$ & 0.012 & $1.82(1.03,3.21)$ & 0.04 \\
\hline Serrated adenoma & $1(0.1 \%)$ & $2(0.3 \%)$ & -- & -- & -- & -- \\
\hline CRC & $2(0.3 \%)$ & $10(1.3 \%)$ & $5.61(1.24,25.39)$ & 0.01 & $7.05(1.35,36.81)$ & 0.02 \\
\hline \multicolumn{7}{|l|}{ Non-neoplastic polyps } \\
\hline Hyperplastic polyps & $60(7.7 \%)$ & $59(7.7 \%)$ & $0.99(0.68,1.44)$ & 0.96 & $0.99(0.67,1.47)$ & 0.96 \\
\hline Inflammatory polyps & $11(1.4 \%)$ & $40(5.2 \%)$ & $3.81(1.94,7.48)$ & $<0.001$ & $4.19(2.08,8.42)$ & $<0.001$ \\
\hline
\end{tabular}

OR-odds ratio; CI-confidence interval; CRC-colorectal cancer.

Table 4 Adjusted odds ratios for the detection of colorectal neoplasia stratified by different characteristics of gastric polyps

\begin{tabular}{|c|c|c|c|c|c|c|}
\hline \multirow[t]{2}{*}{ Subgroup } & \multirow[b]{2}{*}{ No. $(\%)$} & \multicolumn{2}{|l|}{ Advanced adenoma } & \multicolumn{2}{|c|}{ Any adenoma } & \multirow[b]{2}{*}{$P$ value } \\
\hline & & $\begin{array}{l}\text { Adjusted OR } \\
(95 \% \text { CI })\end{array}$ & P value & No. $(\%)$ & $\begin{array}{l}\text { Adjusted OR } \\
(95 \% \mathrm{CI})\end{array}$ & \\
\hline Non-gastric polyps $(n=776)$ & $35(4.5 \%)$ & 1.00 & & $133(17.1 \%)$ & 1.00 & \\
\hline \multicolumn{7}{|l|}{ Gastric polyps } \\
\hline \multicolumn{7}{|l|}{ Histology pattern } \\
\hline FGP $(n=513)$ & $54(10.5 \%)$ & $2.89(1.82,4.58)$ & $<0.001$ & $160(31.2 \%)$ & $2.45(1.85,3.24)$ & $<0.001$ \\
\hline Hyperplastic polyps $(n=166)$ & $20(12.0 \%)$ & $3.16(1.72,5.83)$ & $<0.001$ & $43(25.9 \%)$ & $1.82(1.21,2.77)$ & 0.004 \\
\hline Adenomatous polyps $(n=11)$ & $3(27.3 \%)$ & $7.94(2.02,31.23)$ & 0.013 & $5(45.5 \%)$ & $4.03(1.21,13.40)$ & 0.029 \\
\hline Inflammatory polyps(n=83) & $3(3.6 \%)$ & $0.79(0.24,2.64)$ & 0.49 & $25(30.1 \%)$ & $2.53(1.49,4.29)$ & 0.001 \\
\hline \multicolumn{7}{|l|}{ Number } \\
\hline Single $(n=418)$ & $39(9.3 \%)$ & $2.89(1.82,4.58)$ & $<0.001$ & $125(29.9 \%)$ & $2.44(1.80,3.31)$ & $<0.001$ \\
\hline Multiple (n=352) & $54(10.5 \%)$ & $2.89(1.82,4.58)$ & $<0.001$ & $104(29.5 \%)$ & $2.24(1.64,3.07)$ & $<0.001$ \\
\hline \multicolumn{7}{|l|}{ Size } \\
\hline$<0.5 \mathrm{~cm}(\mathrm{n}=451)$ & $40(8.9 \%)$ & $2.14(1.32,3.48)$ & $<0.001$ & $133(29.5 \%)$ & $2.12(1.59,2.84)$ & $<0.001$ \\
\hline$\geqq 0.5 \mathrm{~cm}(\mathrm{n}=319)$ & $54(10.5 \%)$ & $2.89(1.82,4.58)$ & $<0.001$ & $96(30.1 \%)$ & $2.42(1.75,3.35)$ & $<0.001$ \\
\hline \multicolumn{7}{|l|}{ Location } \\
\hline Gastric body $(n=401)$ & $40(10.0 \%)$ & $2.81(1.71,4.61)$ & $<0.001$ & $120(29.9 \%)$ & $2.33(1.72,3.15)$ & $<0.001$ \\
\hline Fundus $(n=386)$ & $38(9.8 \%)$ & $2.45(1.51,3.98)$ & $<0.001$ & $120(31.1 \%)$ & $2.39(1.77,3.22)$ & $<0.001$ \\
\hline Antrum $(n=108)$ & $12(11.1 \%)$ & $2.99(1.42,6.27)$ & 0.004 & $28(25.9 \%)$ & $1.94(1.18,3.17)$ & 0.009 \\
\hline Others $(\mathrm{n}=55) \#$ & $7(12.7 \%)$ & $4.26(1.71,10.67)$ & 0.002 & $14(25.5 \%)$ & $1.95(1.01,3.78)$ & 0.047 \\
\hline \multicolumn{7}{|l|}{ H. pylori infection } \\
\hline Negative $(n=520)$ & $53(10.2 \%)$ & $2.81(1.77,4.46)$ & $<0.001$ & $147(28.3 \%)$ & $2.26(1.69,3.01)$ & $<0.001$ \\
\hline Positive (n=250) & $24(9.6 \%)$ & $2.40(1.38,4.18)$ & 0.002 & $14(25.5 \%)$ & $2.54(1.80,3.57)$ & $<0.001$ \\
\hline
\end{tabular}

\# including cardia, pylorus and angle. FGP: fundic gastric polyp.

Table 5 Risk of colorectal adenomas in groups with and without gastric polyps according to sex.

\begin{tabular}{|c|c|c|c|c|c|c|}
\hline \multirow[t]{2}{*}{ colorectal neoplasia } & \multirow{2}{*}{$\begin{array}{l}\text { Group with all gastric polyps } \\
\text { OR }(95 \% \mathrm{CI})\end{array}$} & \multicolumn{3}{|c|}{ Group with fundic gland polyps } & \multicolumn{2}{|c|}{ Group with hyperplastic polyps } \\
\hline & & P value & OR $(95 \% \mathrm{CI})$ & P value & OR $(95 \% \mathrm{CI})$ & P value \\
\hline \multicolumn{7}{|l|}{ Any adenoma } \\
\hline Men & $1.87(1.31,2.66)$ & 0.001 & $2.11(1.43,3.11)$ & $<0.001$ & $1.28(0.72,2.30)$ & 0.40 \\
\hline Women & $2.34(1.66,3.29)$ & $<0.001$ & $2.41(1.67,3.50)$ & $<0.001$ & $2.16(1.26,3.71)$ & 0.005 \\
\hline \multicolumn{7}{|l|}{ Advanced adenoma } \\
\hline Men & $1.71(1.01,2.88)$ & 0.04 & $2.04(1.17,3.57)$ & 0.01 & $1.42(0.61,3.28)$ & 0.42 \\
\hline Women & $4.31(2.05,9.02)$ & $<0.001$ & $3.99(1.82,8.74)$ & $<0.001$ & $7.21(2.95,17.63)$ & $<0.001$ \\
\hline \multicolumn{7}{|l|}{ Tubular adenoma } \\
\hline Men & $1.83(1.26,2.65)$ & 0.001 & $1.99(1.32,2.99)$ & 0.001 & $1.37(0.75,2.50)$ & 0.31 \\
\hline Women & $2.32(1.57,3.18)$ & $<0.001$ & $2.32(1.59,3.40)$ & $<0.001$ & $2.08(1.19,3.63)$ & 0.01 \\
\hline
\end{tabular}




\begin{tabular}{|c|c|c|c|c|c|c|}
\hline \multirow[t]{2}{*}{ colorectal neoplasia } & \multicolumn{2}{|l|}{ Group with all gastric polyps } & \multicolumn{2}{|c|}{ Group with fundic gland polyps } & \multicolumn{2}{|c|}{ Group with hyperplastic polyps } \\
\hline & OR $(95 \% \mathrm{CI})$ & P value & OR $(95 \% \mathrm{CI})$ & P value & OR $(95 \% \mathrm{CI})$ & P value \\
\hline \multicolumn{7}{|c|}{ Tubulovillous/villous adenoma } \\
\hline Men & $1.25(0.64,2.43)$ & 0.52 & $1.63(0.81,3.27)$ & 0.17 & $0.51(0.12,2.25)$ & 0.36 \\
\hline Women & $5.10(1.73,15.03)$ & 0.001 & $5.76(1.89,17.52)$ & $<0.001$ & $5.00(1.23,20.35)$ & 0.01 \\
\hline \multicolumn{7}{|l|}{ CRC } \\
\hline Men & $1.57(0.26,9.49)$ & 0.62 & $1.58(2.22,11.30)$ & 0.65 & $2.24(0.2,25.05)$ & 0.50 \\
\hline Women & 1.02(1.01,1.03) & 0.01 & $1.02(1.01,1.04)$ & 0.001 & $1.01(0.99,1.03)$ & 0.03 \\
\hline
\end{tabular}

\# Number of colorectal polyps. OR-odds ratio; CI-confidence interval; CRC-colorectal cancer.

Table 6 Risk of colorectal adenomas in groups with and without gastric polyps according to different age stratifications.

\begin{tabular}{|c|c|c|c|c|c|c|}
\hline \multirow[t]{2}{*}{ Colorectal adenomas } & \multicolumn{2}{|l|}{$\begin{array}{l}\text { Group with } \\
\text { gastric polyps }\end{array}$} & \multicolumn{2}{|l|}{$\begin{array}{l}\text { Group with fundic } \\
\text { gastric polyps }\end{array}$} & \multicolumn{2}{|c|}{ Group with hyperplastic gastric polyps } \\
\hline & OR $(95 \% \mathrm{CI})$ & $P$ value & OR $(95 \%$ CI) & $P$ value & OR (95\% CI) & P value \\
\hline \multicolumn{7}{|l|}{ Any adenoma } \\
\hline $18-29 \mathrm{yr}$ & $3.65(0.38,35.34)$ & 0.24 & $4.67(0.48,45.62)$ & 0.35 & $7.00(0.34,144.06)$ & 0.29 \\
\hline $30-39 \mathrm{yr}$ & $1.44(0.60,3.48)$ & 0.41 & $1.19(0.43,3.28)$ & 0.80 & $1.42(0.28,7.27)$ & 0.48 \\
\hline $40-49$ yr & $1.81(1.02,3.21)$ & 0.04 & $2.31(1.25,4.26)$ & 0.01 & $1.27(0.48,3.38)$ & 0.40 \\
\hline $50-59$ yr & $1.88(1.26,2.81)$ & $<0.001$ & $2.13(1.38,3.31)$ & 0.001 & $1.80(0.95,3.41)$ & 0.05 \\
\hline $60-74$ yr & $2.62(1.73,3.98)$ & $<0.001$ & $2.50(1.59,3.95)$ & $<0.001$ & $1.66(0.85,3.23)$ & 0.10 \\
\hline \multicolumn{7}{|l|}{ Advanced adenoma } \\
\hline $18-29 \mathrm{yr}$ & $1.08(0.97,1.20)$ & 0.50 & $1.10(0.96,1.26)$ & 0.49 & $1.33(0.76,2.35)$ & 0.15 \\
\hline $30-39$ yr & $5.26(0.58,48.06)$ & 0.17 & $5.76(0.58,56.81)$ & 0.13 & $8.00(0.47,138.38)$ & 0.22 \\
\hline $40-49$ yr & $2.87(1.10,7.45)$ & 0.03 & $3.24(1.18,8.89)$ & 0.02 & $1.53(0.30,7.89)$ & 0.40 \\
\hline $50-59$ yr & $1.93(0.98,3.79)$ & 0.05 & $2.33(1.14,4.76)$ & 0.03 & $2.86(1.14,7.19)$ & 0.04 \\
\hline $60-74$ yr & $2.17(1.11,4.23)$ & 0.02 & $1.89(0.90,3.96)$ & 0.13 & $2.65(1.05,6.68)$ & 0.05 \\
\hline
\end{tabular}

OR-odds ratio; CI-confidence interval.

\section{Subgroup analysis}

The increase in colorectal adenoma prevalence in patients with gastric polyps was accordant across all major subgroups based on baseline factors (Supplemental Figure 1). Subgroup analysis suggested no significant interactions in any of the 17 predefined subgroups ( $\mathrm{P}>0.05$ for all comparisons).

\section{Discussion}

The current study prospectively analyzed 1546 consecutive patients and confirmed an association between gastric polyps and colorectal adenomas, especially advanced colorectal adenomas. A significantly higher prevalence of colorectal adenomas (29.7\%) and advanced colorectal adenomas $(10.0 \%)$ were observed in patients with gastric polyps compared to the matched control group $(17.1 \%$ for colorectal adenomas and $4.5 \%$ for colorectal adenomas). Both men and women with gastric polyps had significantly higher prevalence of colorectal adenomas than those of control groups. Furthermore, Subjects over the age of 40 with gastric polyps were more likely to have both advanced and malignant colorectal adenomas.

Although previous studies have shown that FGP $[19,20]$, hyperplastic gastric polyps [21] and gastric adenomas [10, 22] are associated with a higher prevalence of colorectal polyps, most of these were based on retrospective design and limited sample size. The higher prevalence of colorectal adenomas and advanced colorectal adenomas in our cohort is backed up by a multicenter prospective cross-sectional design with large sample size, and particularly, by comprehensive multivariable adjustments and detailed stratification analysis. It should be noted that in this study, patients with gastric polyps had less smoking and alcohol consumption and higher serum cholesterol levels at baseline. This might be due to the reason that we included only age- and sex- matched subjects, which could possibly limit the balance of these baseline risk factors. We also noticed lower $H$. pylori infection rate in the gastric-polyp group at baseline. It is known that $H$. pylori infection are negatively associated with FGP [23] but positively associated with gastric hyperplastic polyps [24]. In this study, we included subjects with different kinds of gastric polyps, with $66 \%$ being FGP, while only $21 \%$ being hyperplastic polyps, thus can explain the lower $H$. pylori infection rate in the gastric-polyp group. Despite this, strong positive correlation between gastric polyps and colorectal adenomas still exists after adjusting the confounding effects of these factors.

The finding that higher prevalence of colorectal adenomas among patients with gastric polyps in this study are of clinical significance, because of the malignancy potential of colorectal adenomas [25]. The majority of CRCs derive from neoplastic adenomatous polyps of the mucosa, among which advanced adenomas are regarded as the clinically relevant precursors [15]. Early removal of these 
polyps before their progression to malignancy can substantially reduce the subsequent incidence and mortality of CRC $[3,26,27]$. The prevalence of CRC is significantly higher among patients with gastric polyps compared with the control group, which is consistent with a previous study [19]. Importantly, $60 \%$ of all colonic adenocarcinomas detected in the gastric polyp group were graded as early-stage cancer and could be endoscopically removed. Early diagnosis of CRC, when tumors are still localized, permits simpler treatment by endoscopic polypectomy or open surgery, which are strongly supported by current guidelines [2, 4, 5]. Based on these results, we highly recommend colonoscopy screening among patients with gastric polyps without alarming symptoms.

Male gender is a recognized risk factor for colorectal neoplasia [28]. We confirmed this finding and found that the presence of gastric polyps in male subjects further raised the prevalence of colorectal adenomas. Another large-scale retrospective study from 2009 demonstrated a higher prevalence of colorectal adenomas in women who had FGP $(\mathrm{OR}=1.43$, 95\% CI: 1.26-1.63) than in men [11]. Here, we found a significant correlation between gastric polyps and colorectal adenomas in both women and men, not only for FGP, but also for all types of gastric polyps, although the risks for women were higher than for men. Women with gastric polyps were more likely to have advanced and malignant colorectal adenomas than men, which was another interesting finding. These results demonstrate that the presence of gastric polyps, either FGPs or gastric hyperplastic polyps, can greatly increase the prevalence of colorectal adenomas among women. Therefore, we also recommend that women with gastric polyps undergo colonoscopy for screening of cancer precursor lesions.

The risk of colorectal neoplasia increases with age [28, 29], and the current guidelines recommend colonoscopy screening in average-risk patients over the age of 50 [4]. Interestingly, patients over 40 with gastric polyps were more likely to have colorectal adenomas than younger patients. Among patients younger than 40 with gastric polyps, prevalence of colorectal adenomas is not significantly increased. Although patients over 50 are already candidates for colonoscopy screening, we recommend that patients with gastric polyps begin screening colonoscopy as early as 40, especially among patients with FGP, since a fair amount of colorectal neoplasms can be detected among this population during the 10-year gap.

The association between gastric and colorectal neoplasia is well clarified in hereditary cancer syndromes, such as familial adenomatous polyposis
(FAP) [30] or hereditary non-polyposis colorectal cancer [31]. However, the precise mechanism underlying the potential relationship between sporadic gastric polyps and colorectal adenomas remains unclear. It is suspected that both genetic and environmental factors play important roles. It has been reported that the genetic alterations found in sporadic gastric and colorectal adenomas are similar [32]. For example, activation of $\beta$-Catenin gene mutations is demonstrated both in the pathogenesis of sporadic FGPs [33] and colorectal adenomas [34]. Specific gene mutations, including adenomatous polyposis coli (APC), Kirsten-ras (K-RAS), and p53, are implicated in both colorectal and gastric tumorigenesis, by driving the progression from healthy epithelia to dysplasia and cancer [35, 36]. Other types of genetic and epigenetic abnormalities, including microsatellite instability and hypermethylation have been associated with primary gastrointestinal cancers [37]. Despite this, the genetic associations between gastric and colorectal polyps are not totally clarified yet, and implementation of next generation sequencing method is warranted to identify novel genetic and epigenetic alterations. The role of environmental factors underlying the gastric-colorectal polyps association remains largely unexplored. Although lifestyle factors such as smoking has been shown to be positively related to both colorectal polyps and gastric polyps [38-40], the mechanism of how these non-genetic factors contributes to the development of both gastric and colorectal polyps merits further studies. Alteration of gut microbiota has been shown to be associated with colorectal adenomas and gastric polyposis[41, 42]. However, controversial evidence has been presented regarding the association of $H$. Pylori infection and colonic neoplasia [43,44]. In our study, the association of gastric polys and colorectal adenomas exists irrespective of the status of $\mathrm{H}$. pylori infection. Therefore, the interactions between $\mathrm{H}$. pylori and other gastric bacteria and their roles in gastric-colorectal polyp co-occurrence remains unknown and merits further studies.

There were a few shortcomings of this study. First, colonoscopy was performed only within six months of EGD. Due to the prolonged formation and slow growing of polyps [4], it is very likely that colorectal polyps were present at the time of EGD. Thus, the risk of de novo colorectal polyp initiation among patients with gastric polyps remains unclear, and long-term follow up study is necessary. Second, although at least six minutes of withdrawal time was required for colonoscopy, we did not measure this. We were unable to eliminate the confounding effect from withdrawal time, since it has been confirmed to 
be associated with increased adenoma detection [45]. Third, due to the very low detection rate of serrated polyps in this study, we were unable to assess the association between gastric polyps and the serrated colorectal polyps, a separate entity with malignant potential [46]. Finally, despite the multicenter advantage of this study, given the geographic variation of colorectal polyps and CRCs among Western and Eastern countries, the generalizability of our results to other regions needs to be further confirmed.

\section{Conclusions}

In summary, our results demonstrate that the presence of gastric polyps, regardless of their number, size, location, or pathology, is an independent risk factor for the detection of colorectal adenomas, particularly advanced adenomas. We recommend screening colonoscopy in patients of both sexes over the age of 40 with gastric polyps. Further studies are required to elucidate the initiation of de novo colorectal polyps among patients with gastric polyps, the cost effectiveness of such a colonoscopy screening approach, the application of our results to Western countries, and the clarification of the precise mechanism underlying the close relationship between gastric and colorectal polyps.

\section{Supplementary Material}

Supplementary figures and tables. http://www.jcancer.org/v10p4623s1.pdf

\section{Abbreviations}

CRC, colorectal cancer; FGP, fundic gland polyp; EGD, esophagogastroduodenoscopy; BMI, body mass index; NSAIDS, non-steroidal anti-inflammatory drugs; OR, odds ratio; $\mathrm{CI}$, confidence interval.

\section{Acknowledgements}

We thank all the patients, doctors, pathologists, and nurses for their friendly participation. We thank Futian Luo for his assistance with statistical analyses.

\section{Author Contributions}

Guarantor of the article: $\mathrm{CMH}, \mathrm{ZSH}$; Study concept and design: ZSH, ZDP, YZW, HLR, NSC, LMR, HSS, WSB, LL, LMY, ZHS, LJH, ZBH, CY, $\mathrm{CMH}$; acquisition of data: $\mathrm{ZSH}, \mathrm{ZDP}, \mathrm{CY}, \mathrm{CMH}$; analysis and interpretation of data: ZSH, ZDP, YZW, HLR, CY, CMH; drafting of the manuscript: ZSH, ZDP; critical revision of the manuscript for important intellectual content: ZSH, ZDP, YZW, HLR, NSC, LMR, HSS, WSB, LL, LMY, ZHS, ZBH, CY, CMH; statistical analysis: ZSH, ZDP, YZW, HLR, CY, CMH.
All authors approved the final version of the manuscript.

\section{Funding}

This project was supported by grant from Science and technology innovation young talents of Guangdong special plan (2016TQ03R296). The funders had no role in study design, data collection and analysis, decision to publish, or preparation of the manuscript.

\section{Ethics and consent to participate}

The study was conducted and reported according to the study protocol, which was approved by Ethics Review Committees at each study center (The First Affiliated Hospital, Sun Yat-sen University; Meizhou People's Hospital; Shantou Central Hospital; 2012[NO.32]). Written informed consents were obtained from participants upon their arrival at the study clinic and before the procedures.

\section{Data availability statements}

The datasets used and/or analyzed during the current study available from the corresponding author on reasonable request.

\section{Competing Interests}

The authors have declared that no competing interest exists.

\section{References}

1. Regula J, Rupinski M, Kraszewska E, Polkowski M, Pachlewski J, Orlowska J, et al. Colonoscopy in colorectal-cancer screening for detection of advanced neoplasia. The New England journal of medicine. 2006; 355: 1863-72.

2. Lieberman DA, Rex DK, Winawer SJ, Giardiello FM, Johnson DA, Levin TR. Guidelines for colonoscopy surveillance after screening and polypectomy: a consensus update by the US Multi-Society Task Force on Colorectal Cancer. Gastroenterology. 2012; 143: 844-57.

3. Zauber AG, Winawer SJ, O'Brien MJ, Lansdorp-Vogelaar I, van Ballegooijen $\mathrm{M}$, Hankey BF, et al. Colonoscopic polypectomy and long-term prevention of colorectal-cancer deaths. The New England journal of medicine. 2012; 366: 687-96.

4. Winawer S, Fletcher R, Rex D, Bond J, Burt R, Ferrucci J, et al. Colorectal cancer screening and surveillance: clinical guidelines and rationale-Update based on new evidence. Gastroenterology. 2003; 124: 544-60.

5. Levin B, Lieberman DA, McFarland B, Smith RA, Brooks D, Andrews KS, et al. Screening and surveillance for the early detection of colorectal cancer and adenomatous polyps, 2008: a joint guideline from the American Cancer Society, the US Multi-Society Task Force on Colorectal Cancer, and the American College of Radiology. CA: a cancer journal for clinicians. 2008; 58 : 130-60.

6. Chiu HM, Ching JY, Wu KC, Rerknimitr R, Li J, Wu DC, et al. A Risk-Scoring System Combined With a Fecal Immunochemical Test Is Effective in Screening High-Risk Subjects for Early Colonoscopy to Detect Advanced Colorectal Neoplasms. Gastroenterology. 2016; 150: 617-25.e3.

7. Park DI, Park SH, Yoo TW, Kim HS, Yang SK, Byeon JS, et al. The prevalence of colorectal neoplasia in patients with gastric cancer: a Korean Association for the Study of Intestinal Disease (KASID) Study. J Clin Gastroenterol. 2010; 44: $102-5$.

8. Yoo HM, Gweon TG, Seo HS, Shim JH, Oh SI, Choi MG, et al. Role of preoperative colonoscopy in patients with gastric cancer: a case control study of the prevalence of coexisting colorectal neoplasms. Annals of surgical oncology. 2013; 20: 1614-22

9. Wu ZJ, Lin Y, Xiao J, Wu LC, Liu JG. Clinical significance of colonoscopy in patients with upper gastrointestinal polyps and neoplasms: a meta-analysis. PloS one. 2014; 9: e91810.

10. Cappell MS, Fiest TC. A multicenter, multiyear, case-controlled study of the risk of colonic polyps in patients with gastric polyps. Are gastric adenomas a 
new indication for surveillance colonoscopy? J Clin Gastroenterol. 1995; 21: 198-202.

11. Genta RM, Schuler CM, Robiou CI, Lash RH. No association between gastric fundic gland polyps and gastrointestinal neoplasia in a study of over 100,000 patients. Clinical gastroenterology and hepatology : the official clinical practice journal of the American Gastroenterological Association. 2009; 7: $849-54$.

12. Cimmino DG, Mella JM, Luna P, Gonzalez R, Pereyra L, Fischer C, et al. Risk of colorectal polyps in patients with sporadic gastric polyps: A case-control study. World journal of gastrointestinal endoscopy. 2013; 5: 240-5.

13. Patel K, Hoffman NE. The anatomical distribution of colorectal polyps at colonoscopy. J Clin Gastroenterol. 2001; 33: 222-5.

14. Hamilton S.R. Aaltonen L.A (eds). World Health Organization Classification of Tumours. Pathology and Genetics of Tumours of the Digestive System. Lyon, France: IARC Press; 2000.

15. Brenner H, Hoffmeister M, Stegmaier C, Brenner G, Altenhofen L, Haug U. Risk of progression of advanced adenomas to colorectal cancer by age and sex: estimates based on 840,149 screening colonoscopies. Gut. 2007; 56: 1585-9.

16. Bond JH. Polyp guideline: diagnosis, treatment, and surveillance for patients with colorectal polyps. Practice Parameters Committee of the American College of Gastroenterology. The American journal of gastroenterology. 2000; 95: 3053-63.

17. Sobin LH GM, Wittekind C. TNM classification of malignant tumours: John Wiley \& Sons; 2011.

18. Chung SJ, Kim YS, Yang SY, Song JH, Kim D, Park MJ, et al. Five-year risk for advanced colorectal neoplasia after initial colonoscopy according to the baseline risk stratification: a prospective study in 2452 asymptomatic Koreans. Gut. 2011; 60: 1537-43.

19. Teichmann J, Weickert U, Riemann JF. Gastric fundic gland polyps and colonic polyps - is there a link, really? European journal of medical research. 2008; 13: $192-5$.

20. Jung A, Vieth M, Maier O, Stolte M. Fundic gland polyps (Elster's cysts) of the gastric mucosa. A marker for colorectal epithelial neoplasia? Pathology, research and practice. 2002; 198: 731-4.

21. Cao H, He N, Song S, Xu M, Piao M, Yan F, et al. Is surveillance colonoscopy necessary for patients with sporadic gastric hyperplastic polyps? PloS one. 2015; 10: e0122996.

22. Yang $\mathrm{MH}$, Son HJ, Lee JH, Kim MH, Kim JY, Kim YH, et al. Do we need colonoscopy in patients with gastric adenomas? The risk of colorectal adenoma in patients with gastric adenomas. Gastrointestinal endoscopy. 2010; 71: 774-81.

23. Carmack SW, Genta RM, Schuler CM, Saboorian MH. The current spectrum of gastric polyps: a 1-year national study of over 120,000 patients. The American ournal of gastroenterology. 2009; 104: 1524-32.

24. Elhanafi S, Saadi M, Lou W, Mallawaarachchi I, Dwivedi A, Zuckerman M, et al. Gastric polyps: Association with Helicobacter pylori status and the pathology of the surrounding mucosa, a cross sectional study. World journal of gastrointestinal endoscopy. 2015; 7: 995-1002.

25. Fenoglio CM, Pascal RR. Colorectal adenomas and cancer: pathologic relationships. Cancer. 1982; 50: 2601-8

26. Winawer SJ, Zauber AG, Ho MN, O'Brien MJ, Gottlieb LS, Sternberg SS, et al. Prevention of colorectal cancer by colonoscopic polypectomy. The National Polyp Study Workgroup. The New England journal of medicine. 1993; 329: 1977-81.

27. Loberg M, Kalager M, Holme O, Hoff G, Adami HO, Bretthauer M. Long-term colorectal-cancer mortality after adenoma removal. The New England journal of medicine. 2014; 371: 799-807.

28. Wong MC, Lam TY, Tsoi KK, Hirai HW, Chan VC, Ching JY, et al. A validated tool to predict colorectal neoplasia and inform screening choice for asymptomatic subjects. Gut. 2014; 63: 1130-6.

29. Ries L AG MD, Krapcho M, et al. SEER cancer statistics review, 1975-2005.: Bethesda, MD: 2008.

30. Wood LD, Salaria SN, Cruise MW, Giardiello FM, Montgomery EA. Upper GI tract lesions in familial adenomatous polyposis (FAP): enrichment of pyloric gland adenomas and other gastric and duodenal neoplasms. The American journal of surgical pathology. 2014; 38: 389-93

31. Gylling A, Abdel-Rahman WM, Juhola M, Nuorva K, Hautala E, Jarvinen HJ, et al. Is gastric cancer part of the tumour spectrum of hereditary non-polyposis colorectal cancer? A molecular genetic study. Gut. 2007; 56: 926-33.

32. Abraham SC, Park SJ, Lee JH, Mugartegui L, Wu TT. Genetic alterations in gastric adenomas of intestinal and foveolar phenotypes. Modern pathology : an official journal of the United States and Canadian Academy of Pathology, Inc. 2003; 16: 786-95

33. Abraham SC, Nobukawa B, Giardiello FM, Hamilton SR, Wu TT. Sporadic fundic gland polyps: common gastric polyps arising through activating mutations in the beta-catenin gene. The American journal of pathology. 2001; 158: 1005-10.

34. Samowitz WS, Powers MD, Spirio LN, Nollet F, van Roy F, Slattery ML. Beta-catenin mutations are more frequent in small colorectal adenomas than in larger adenomas and invasive carcinomas. Cancer research. 1999; 59: 1442-4.

35. Hamilton JP, Meltzer SJ. A review of the genomics of gastric cancer. Clinical gastroenterology and hepatology : the official clinical practice journal of the American Gastroenterological Association. 2006; 4: 416-25.

36. Smith G, Carey FA, Beattie J, Wilkie MJ, Lightfoot TJ, Coxhead J, et al. Mutations in APC, Kirsten-ras, and p53--alternative genetic pathways to colorectal cancer. Proceedings of the National Academy of Sciences of the United States of America. 2002; 99: 9433-8

37. Yamashita K, Arimura Y, Kurokawa S, Itoh F, Endo T, Hirata K, et al. Microsatellite instability in patients with multiple primary cancers of the gastrointestinal tract. Gut. 2000; 46: 790-4.

38. Di Giulio E, Lahner E, Micheletti A, Milione M, D'Ambra G, Bordi C, et al. Occurrence and risk factors for benign epithelial gastric polyps in atrophic body gastritis on diagnosis and follow-up. Aliment Pharmacol Ther. 2005; 21: 567-74

39. Wang FW, Young SC, Chen RY, Lin $\mathrm{KH}$, Chen YH, Hsu PI, et al. The Prevalence and Risk Factors of Gastric Polyp in Asymptomatic Patients Receiving Health Examination. Gastroenterology research and practice. 2018; 2018: 9451905.

40. Botteri E, Iodice S, Raimondi S, Maisonneuve P, Lowenfels AB. Cigarette smoking and adenomatous polyps: a meta-analysis. Gastroenterology. 2008; 134: 388-95.

41. Dulal S, Keku TO. Gut microbiome and colorectal adenomas. Cancer journal (Sudbury, Mass). 2014; 20: 225-31.

42. Ren R, Wang Z, Sun H, Gao X, Sun G, Peng L, et al. The gastric mucosal-associated microbiome in patients with gastric polyposis. Scientific reports. 2018; 8: 13817.

43. Shmuely H, Passaro D, Figer A, Niv Y, Pitlik S, Samra Z, et al. Relationship between Helicobacter pylori CagA status and colorectal cancer. The American journal of gastroenterology. 2001; 96: 3406-10.

44. Moss SF, Neugut AI, Garbowski GC, Wang S, Treat MR, Forde KA. Helicobacter pylori seroprevalence and colorectal neoplasia: evidence against an association. Journal of the National Cancer Institute. 1995; 87: 762-3.

45. Barclay RL, Vicari JJ, Doughty AS, Johanson JF, Greenlaw RL. Colonoscopic withdrawal times and adenoma detection during screening colonoscopy. The New England journal of medicine. 2006; 355: 2533-41.

46. Noffsinger AE. Serrated polyps and colorectal cancer: new pathway to malignancy. Annu Rev Pathol. 2009; 4: 343-64. 